\title{
Load Balancing Model using Elastic Technique in Cloud Computing
}

\author{
Sovban Nisar, Deepika Arora
}

\begin{abstract}
A structural design in which virtual machines are implicated and connect to the cloud service provider is called cloud computing. On the behalf of the users, the virtual machines connect to the cloud service provider. The uncertainties overload the virtual machines. The genetic algorithm is implemented for the migration of virtual machine in the earlier study. The genetic algorithm is low depicts latency within the network is high at the time of virtual machine migration. The genetic algorithm is implemented for virtual machine migration in this study. The proposed algorithm is applied in MATLAB in this work. The obtained results are compared with the results of earlier algorithm. Various parameters like latency, bandwidth consumption, and space utilization are used to analyze the achieved results.
\end{abstract}

Keywords: MATLAB, virtual machine migration.

\section{INTRODUCTION}

The cloud computing can be described as an on-demand service pool which connects various servers to each other for providing services to aiming clients. The cloud providers may contain direct access to these services. Therefore, the resources can be used according to the requirement. The user can extract and modifies the data stored in the clouds. The different services to the user are provided on demand using a feature called "cloud service provider". This trait makes certain that the amount of services being utilized for any number of times can be employed for calculating the expense of the user to access that service. The cloud computing system provides extremely complicated applications in different environments. In addition, some skilled concentrated services are provided in each environment. In cloud computing, common group of resources is provided to the users. Using cloud computing, the users can utilize these resources according to their need everywhere. The main objective of this technology is to maintain the minimum cost to access the services. It is analyzed that the software and hardware assets obtained using internet remain present in the virtual system and supports to provide the services. The user accesses a common group of resources using cloud computing on the basis of demand. The virtualization allows user to subscribe and use the services for a certain time period by getting access of the common group of resources using cloud computing.

Manuscript received on April 10, 2021.

Revised Manuscript received on April 16, 2021.

Manuscript published on April 30, 2021.

* Correspondence Author

Sovban Nisar*, M.Tech. Department of Computer Science and Engineering, Geeta Engineering College, Naultha, Panipat, India

Deepika Arora, Asst. Prof. Department of Computer Science and Engineering, Naultha, Panipat, India

(C) The Authors. Published by Blue Eyes Intelligence Engineering and Sciences Publication (BEIESP). This is an open access article under the CC BY-NC-ND license (http://creativecommons.org/licenses/by-nc-nd/4.0/)
The cloud computing reduces application cost reduced and allows the user to access more hardware parts [1].The information regarding physical place or the design of the system is not required in this technology. Some of the significant aspects of cloud computing are identified as the geographic allocation-based service orientation, homogeneity,

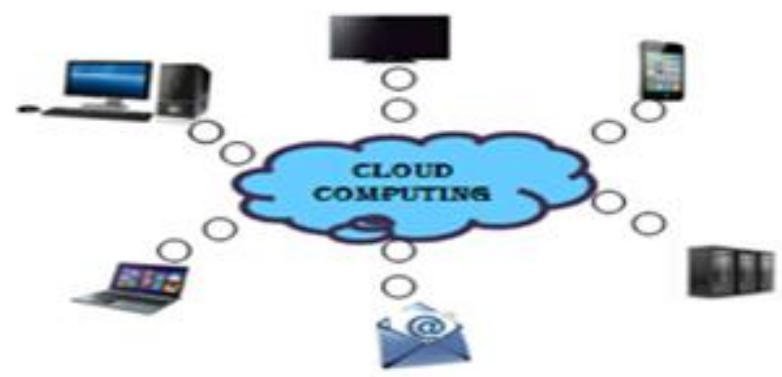

Fig. 1.1 Cloud Computing Environment

virtualization, current security and inexpensive software. In cloud computing, the user can use applications without installing. The user can also manage and get the access of the private files at any location just using internet. In cloud computing, the centralized storage aspect provides competent bandwidth, memory, computing and resource distribution. The Fig. 1.1 drawn below gives a general idea of cloud computing environment.

Some fundamental requirements of framework, hardware and software are needed for the designing of a cloud. Some fundamental steps followed during the designing of the cloud are described below:

- Grid Computing: The grid computing uses parallel computing to solve the big scale issues.

- Utility computing: The clients should be charged on the basis of the amount of services accessed and the time period for which these services are accessed. The utility computing includes resources utilized for these services.

- SAAS: The SaaS is identified as a part of service in cloud computing system. This system provides networkbased subscription for the applications.

- Cloud Computing: The cloud computing system involves all services provided by the above-mentioned systems. This implies that the resources can be accessed by the user at any time from any place [2].

\section{RESEARCH METHODOLOGY}

For handling the problems related to node failure in cloud networks, an algorithm named BFO is used in this research. Several nodes are included in a proposed algorithm.

Published By: 


\section{Load Balancing Model using Elastic Technique in Cloud Computing}

Depending upon the failure rate and minimal execution time, a participant node is chosen among all these nodes. In this scenario, the threshold value is fixed using the master node. There are two parameters included in this threshold value. The master node chooses nodes having equivalent or less failure rate with least execution time as the participant nodes. In comparison to threshold value, the value of node $\mathrm{N} 1$ is less. Thus, the selection of this node is carried out as a candidate or participant node. There is a less and a higher parameter included in node N2. Therefore, it is not possible to select this node as candidate node. A node N3 is elected as candidate node since it includes the value equivalent to threshold. It is not possible to choose another node N4 as participant node because its value is higher as compared to that of the threshold level. After it is selected, the candidate node starts its functioning. In this scenario, various tasks are initiated. One node switch to other location once the task is completed. Thus, task failure occurs as a result. For remove the issue of failure arising because of node mobility, a new methodology is proposed. In the new algorithmic approach, a novel parametric quality known as master node time is included. The ultimate time connecting the end clients is known as master node time which helps in performing node cooperation.

\section{Code of proposed system}

\section{Begin}

Input: Virtual machine

Output: Task migration

Define Number of Tasks as Tk

Threshold value of failure rate as FR

Threshold value of execution time as ER

Repeat while virtual machine is selected for the Task (Tk)

If (FR of machine $i>$ FR of machine $\mathrm{i}+1$ )

If (ER of machine $i>$ ER of machine $i+1)$

Select $i+1$ as best machine

End if

End if

End of while

If (virtual i get overloaded=true)

Calculate weight ()

If (weigh of $i>$ weight of $i+1$ )

Select machine i for migration

Else

Select execute weight algorithm

End if

\section{EXPERIMENTAL RESULTS}

This work uses MATLAB as a simulation tool for implementing the new algorithm since in real time scenarios, its complexity is high. Based on power usage and execution time, the comparison among the performance of proposed and existing algorithms is evaluated. Table 1 shows the simulation parameters used in this research.

Table 3.1: Simulation Parameters

\begin{tabular}{|l|l|}
\hline Number of VM & 10 \\
\hline Number of cloudlets & 60 \\
\hline Host Memory & 2 GB \\
\hline Processor & Xenon \\
\hline Number of Data centers & 5 \\
\hline
\end{tabular}

On which task will be migrated for the execution.

\subsection{Comparison graph of Response Time}

Fig. 3.2 depicts the response time-based comparison between improved GA and new enhanced improved GA so that the performance of both of these algorithms can be analysed. The new algorithm is superior as its response time is lesser to its counterpart.

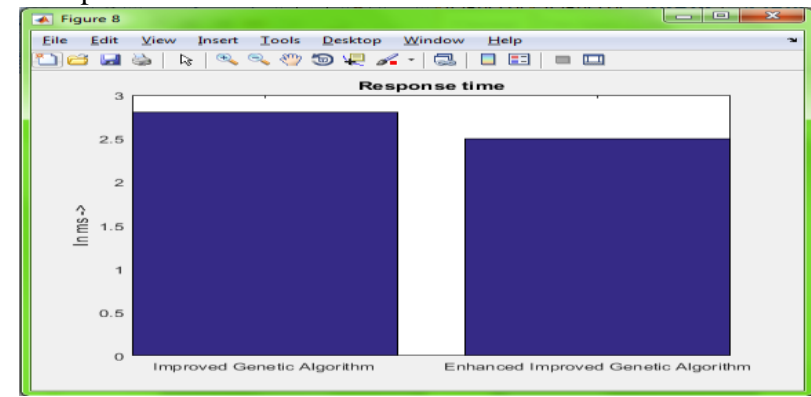

Fig.3.2 Comparison graph of Response Time

\subsection{Comparison graph of Finish Time}

Fig. 3.3 depicts the finish time-based comparison between improved GA and new enhanced improved GA so that the performance of both of these algorithms can be analyzed. The new algorithm is superior as its finish time is lesser to its counterpart.

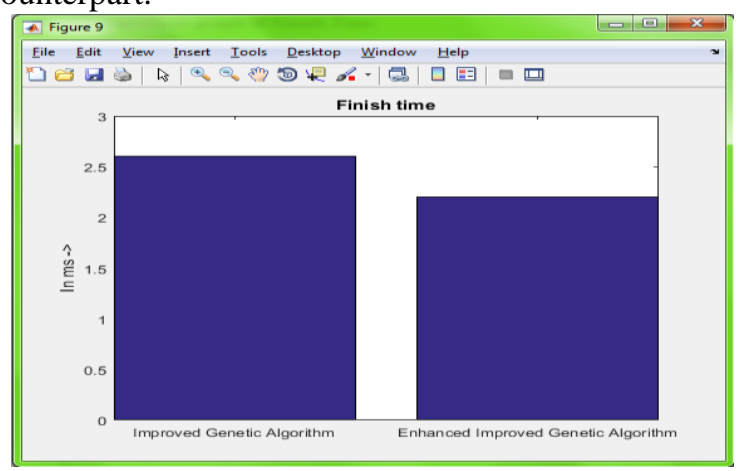

Fig.3.3 Comparison graph of Finish Time

\subsection{Comparison graph of Energy Consumption}

Fig. 3.4 compares improved GA and new enhanced improved GA on the basis of energy consumed by them. The new algorithm is superior as it consumes lesser energy than its counterpart.

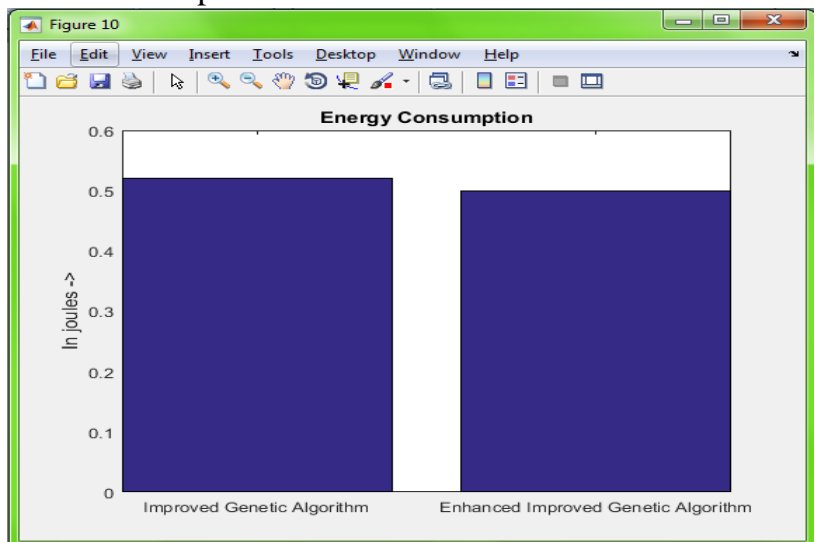

Fig.3.4 Comparison graph of Energy Consumption

Published By:

Blue Eyes Intelligence Engineering \& Sciences Publication

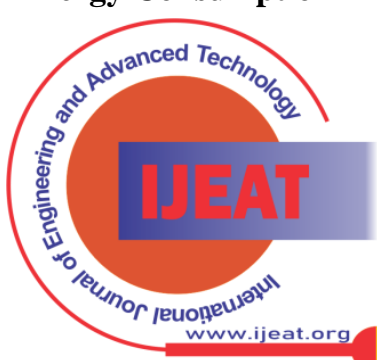

Exploring Innovation 


\subsection{Comparison graph of Cost}

Fig. 3.5 depicts the cost-based comparison between improved GA and new enhanced improved GA so that the performance of both of these algorithms can be analysed. The new algorithm is superior as its cost is lesser to its counterpart.

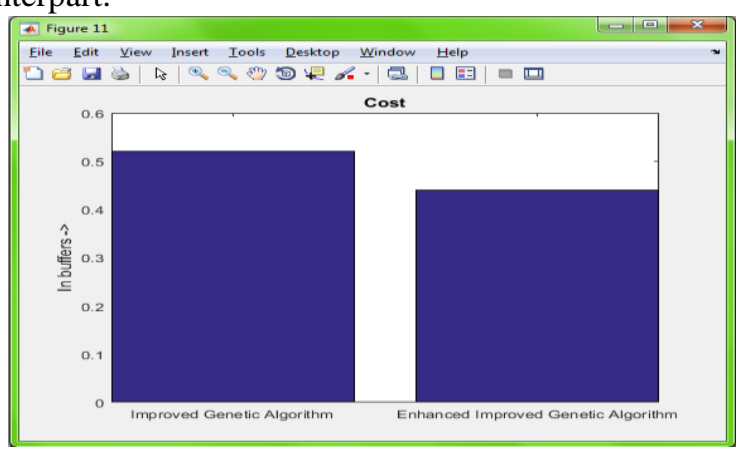

Fig.3.5 Comparison graph of Cost

\subsection{Comparison graph of Number of Migrations}

Fig. 3.6 compares improved GA and new enhanced improved GA on the basis of no. of migrations. The new algorithm is superior to its counterpart with lesser number of migrations.

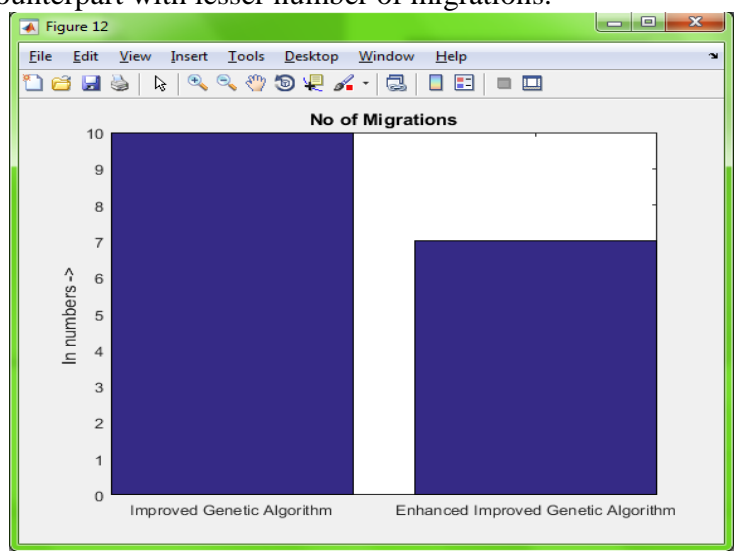

Fig.3.6 Comparison graph of Number of Migrations

\subsection{Overall comparison}

a) Qualitative Comparison of Improved GA and

\section{Presented Enhanced Improved GA -}

Table 3.7 shows the qualitative comparison of improved GA and presented enhanced improved GA to measure the performance of both of these algorithms. The newly devised GA is fast, reliable, has less chances of fault in the network and is less complex for cloudlet assignment.

Table 3.7 Qualitative comparison of the algorithms

\begin{tabular}{|l|l|l|}
\hline \multicolumn{1}{|c|}{ Parameters } & \multicolumn{1}{|c|}{$\begin{array}{c}\text { Improved Genetic } \\
\text { Algorithm }\end{array}$} & $\begin{array}{c}\text { Proposed Enhanced } \\
\text { Improved Genetic Algorithm }\end{array}$ \\
\hline $\begin{array}{l}\text { Cloud } \\
\text { Assignment }\end{array}$ & $\begin{array}{l}\text { Complex procedure for the } \\
\text { cloudlet assignment. }\end{array}$ & $\begin{array}{l}\text { Less complex procedure for } \\
\text { the cloudlet assignment. }\end{array}$ \\
\hline Strength & $\begin{array}{l}\text { The algorithm is slow as } \\
\text { compared to enhanced } \\
\text { improved genetic algorithm. }\end{array}$ & $\begin{array}{l}\text { The algorithm is fast for the } \\
\text { virtual machine migration than } \\
\text { the improved genetic } \\
\text { algorithm. }\end{array}$ \\
\hline Chances of fault & High chances of fault & Less chances of fault \\
\hline Cost & $\begin{array}{l}\text { Load Balancing is } \\
\text { expensive }\end{array}$ & Load Balancing is cheaper \\
\hline
\end{tabular}

b) Quantitative Comparison of Improved GA and

Proposed Enhanced Improved GA -

Table 3.8 depicts the improved genetic and proposed enhanced improved genetic algorithm comparison on the

basis of some metrics including response time, finish time, energy consumption, cost and no of migrations. After analyzing the table, it appears that the presented algorithm outperforms the old algorithm in regard to all considered metrics.

Table 3.8 Quantitative comparison of the parameters

\begin{tabular}{|l|c|c|}
\hline \multicolumn{1}{|c|}{ Parameter } & $\begin{array}{c}\text { Improved Genetic } \\
\text { Algorithm }\end{array}$ & $\begin{array}{c}\text { Proposed Enhanced } \\
\text { Improved Genetic } \\
\text { Algorithm }\end{array}$ \\
\hline Response Time & 2.8 seconds & 2.5 seconds \\
\hline Finish Time & 2.9 seconds & 2.2 seconds \\
\hline $\begin{array}{l}\text { Energy } \\
\text { Consumption }\end{array}$ & 0.57 joules & 0.5 joules \\
\hline Cost & 0.56 buffers & 0.45 buffers \\
\hline No of migration & 10 & 7 \\
\hline
\end{tabular}

\section{CONCLUSION}

There are two broader categories of load balancing algorithms known as dynamic and static. The decision related to load shifting is not based on the existing status of scheme in the static type of algorithms. Awareness related to the resources and applications of system are required here. It is possible to determine the efficiency of VMs once the task is assigned. Based on their functioning, the master node assigns tasks to other slave nodes. Therefore, the assigned work is processed by the slave processors and the master process delivers the achieved outcomes. To make decisions related to load balancing the current system status is used by dynamic algorithms. Thus, load shifting occurs as per the existing system status. For a faster implementation, the procedures are allowed to shift from an overused method to an underused method in an animated manner. This research resolves the load balancing issue being faced in the cloud architectures. In the systems, latency can be increased due to load balancing. For performing VM migration, the work carried out in the past has applied GA. It is seen through this research that the complexity of genetic algorithm is high. Thus, the time of virtual machine migration is increased. This research work aims to perform virtual machine migration by applying improved Genetic technique. MATLAB is employed for implementing the newly devised algorithm and several parametric values are calculated for analysing the efficiency of this algorithmic approach. The outcomes show that in comparison to existing algorithmic approach, the new algorithm is better.

\section{REFERENCES}

1. Randles, M.; Lamb, D.; Taleb-Bendiab, A., "A Comparative Study into Distributed Load Balancing Algorithms for Cloud Computing", IEEE 24th International Conference on Advanced Information Networking and Applications Workshops (WAINA), April 2010, pp.551-556.

Published By:

Blue Eyes Intelligence Engineering

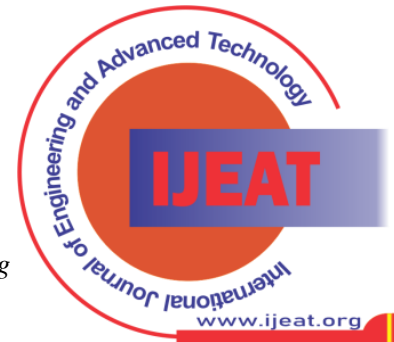




\section{Load Balancing Model using Elastic Technique in Cloud Computing}

2. Fang, Yiqiu, Fei Wang, and Junwei Ge, "A task scheduling algorithm based on load balancing in cloud computing", Web Information Systems and Mining. Springer Berlin Heidelberg, 23 October 2010, Pages 271-277.

3. Chaczko, Zenon, Venkatesh Mahadevan, ShahrzadAslanzadeh and Christopher Mcdermid, "Availability and load balancing in cloud computing", International Conference on Computer and Software Modeling, Singapore, Vol. 14, 2011.

4. Samrat Kumar Dey, Md. Raihan Uddin, Kh. Mohaimenul Kabir and Md. Mahbubur Rahman, "Enhancing the Security of Cloud Computing: Genetic Algorithm and QR Code Approach", Proceedings of the 2017 4th International Conference on Advances in Electrical Engineering, 2017.

5. Shui Han, Jianchuan Xing, "Ensuring Data Storage Through A Novel Third Party Auditor Scheme in Cloud Computing", IEEE computer science \& Technology, 2011 pp 264-268.

6. Sean Carlin, Kevin Curran, "Cloud Computing Security", International Journal of Ambient Computing and Intelligence, January 2011, pp 14-19.

7. Peter Mell, Timothy Grance, "The NIST Definition of Cloud Computing", Recommendations of the National Institute of Standards and Technology, September 2011, Special Publication 800-145

8. Dr Nashaat el-Khameesy, Hossam Abdel Rahman, "A Proposed Model for Enhancing Data Storage Security in Cloud Computing Systems", 2012, vol-3

9. Arora Pankaj, Wadhawan C. Rubal, Er. Ahuja P. Satinder, "Cloud Computing Security Issue in Infrastructure as a Service", International Journal of Advance Research in Computer Science and Software Engineering, 2012

10. Habib, S. M., Hauke, S., \&Ries, S., "Trust as a facilitator in cloud computing: a survey", Journal of Cloud Computing, 2012, page 0118.

11. WANG Bei, LI Jun, "Load Balancing Task Scheduling based on Multi-Population Genetic Algorithm in Cloud Computing", Proceedings of the 35th Chinese Control Conference, July 2016, pp 27-29.

12. Srinivas.J, K. Venkata Subba Reddy, Dr. A. MoizQyser, "Cloud Computing Basics", International journal of advanced research in computer and communication engineering, 2012, pp. 343-347.

\section{AUTHORS PROFILE}

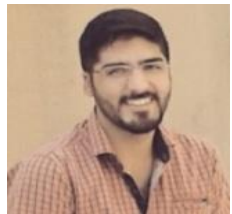

Sovban Nisar, is M.Tech Scholar in the Department of Computer Science and Engineering at Geeta Engineering College, Naultha, Panipat, India. He has done B.Tech. from Kurukshetra University, India. His areas of interest are Cloud Computing. Data Science and Big Data.

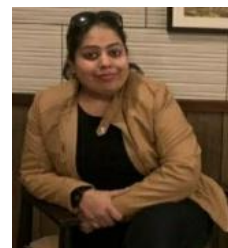

Deepika Arora, is Assistant Professor in Department of Computer Science and Engineering, Geeta Engineering College, Panipat, She has Qualified M. Tech. (CSE) from M.M. University, Mullana (Ambala) and She is perusing $\mathrm{PhD}$ from M.M. University. Her areas of interest are Big Data, Adhoc Networks and Cloud Computing.

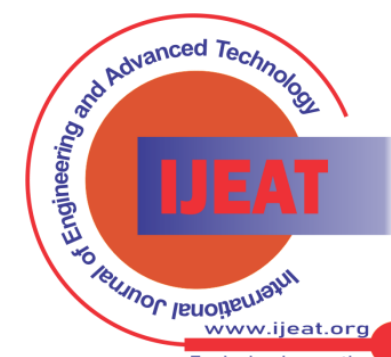

